\title{
Cobalt-Electrocatalytic Hydrogen Atom Transfer for Functionalization of Unsaturated C-C Bonds
}

Authors: Samer Gnaim ${ }^{1 \#}$, Adriano Bauer ${ }^{1 \#}$, Hai-Jun Zhang ${ }^{1 \#}$, Longrui Chen ${ }^{1}$, Cara Gannet ${ }^{4}$, Christian A. Malapit $^{3}$, David Hill ${ }^{2}$, David Vogt ${ }^{3}$, Tianhua Tang ${ }^{3}$, Ryan Daley ${ }^{1}$, Wei Hao ${ }^{1}$, Mathilde Quertenmont ${ }^{5}$, Wesley D. Beck ${ }^{3}$, Elya Kandahari ${ }^{2}$, Julien C. Vantourout ${ }^{1}$, Pierre-Georges Echeverria ${ }^{5}$, Hector Abruna ${ }^{4 *}$, Donna Blackmond $^{1 *}$, Shelley Minteer ${ }^{3 *}$, Sarah Reisman ${ }^{2 *}$, Matthew S. Sigman ${ }^{3 *}$, Phil S. Baran ${ }^{1 *}$

\author{
Affiliations: \\ ${ }^{1}$ Department of Chemistry, The Scripps Research Institute (TSRI), 10550 North Torrey Pines Road, La Jolla, \\ CA 92037, USA.
}

${ }^{2}$ The Warren and Katharine Schlinger Laboratory for Chemistry and Chemical Engineering, Division of Chemistry and Chemical Engineering, California Institute of Technology, Pasadena, California 91125, United States.

${ }^{3}$ Department of Chemistry, University of Utah, 315 South 1400 East, Salt Lake City, Utah 84112, United States.

${ }^{4}$ Department of Chemistry and Chemical Biology, Cornell University, Ithaca, New York 14853, United States

${ }^{5}$ Minakem Recherche, 145 Chemin des Lilas, 59310 Beuvry-la-Forêt, France.

The study and application of transition metal hydrides (TMH) has been an active area of chemical research since the early 1960 's. ${ }^{1}$ The use of TMHs has been broadly bifurcated into fields focused on energy storage through the reduction of protons to generate hydrogen ${ }^{2,3}$ and in organic synthesis for the functionalization of unsaturated $\mathrm{C}-\mathrm{C}, \mathrm{C}-\mathrm{O}$, and $\mathrm{C}-\mathrm{N}$ bonds. ${ }^{4,5}$ In the former instance, electrochemical means for driving such reactivity has been commonplace since the 1950 's. ${ }^{6}$ In contrast, the use of stoichiometric exogenous organic and metal-based reductants to harness the power of TMHs in synthetic chemistry remains the norm. In particular, Co-based TMHs have found widespread use for the derivatization of olefins and alkynes in complex molecule construction, often via a net hydrogen atom transfer (HAT). ${ }^{7}$ Here, we show how an electrocatalytic approach inspired by decades of energy storage precedent can be leveraged in the context of modern organic synthesis. Such an approach not only offers benefits in terms of sustainability and efficiency but also enables enhanced chemoselectivity and unique and tunable reactivity. Ten different reaction manifolds across dozens of substrates are thus exemplified, along with a detailed mechanistic and computational analysis of this scalable electrochemical entry into Co-H chemistry.

TMH species have been a vibrant topic for exploration in both organic and organometallic synthesis. ${ }^{1,8}$ Pioneering studies in this field have led to a deep understanding of metal hydrides ${ }^{9}$ that has allowed synthetic chemists to establish these species as selective mediators for hydrogen atom transfer 
(HAT) chemistry. ${ }^{7,10}$ Such insights have led to the discovery of unique selectivity for known transformations ${ }^{11}$ along with the development of new chemical reactivity. ${ }^{12}$ HAT, the concerted migration of a proton and an electron from a TM-H bond to an acceptor molecule, has emerged as one of the most useful chemical processes for the hydrofunctionalization of alkenes. ${ }^{4}$ In its most common manifestation, the generation of a TMH involves the exposure of an appropriate metal complex to a stoichiometric amount of reductant, such as a silane. Its subsequent interaction with an olefin leads to the formation of a $\mathrm{C}-\mathrm{H}$ bond at the less electronically stabilized position along with a carbon-centred radical on the adjacent position. This intermediate can then be trapped with various agents to form new $\mathrm{C}-\mathrm{C}, \mathrm{C}-\mathrm{N}, \mathrm{C}-$ $\mathrm{O}$, and $\mathrm{C}-\mathrm{X}$ bonds. ${ }^{13-17}$ While the overall process formally requires only the addition of a single proton and an electron to form the active TMH catalytic species, exogenous chemical oxidants, such as peroxides and $\mathrm{N}$-fluoropyridinium salts, are often required to illicit this reactivity. The application of HAT chemistry on a large scale could be problematic due to the need for an excess amount of external reductants with or without peroxide additives, resulting in poor atom economy and scalability concerns (Figure 1A). ${ }^{18}$ Given the growing documented utility of such reactions in organic synthesis, it is clear that more practical and universal variants are needed. ${ }^{19,20}$

In parallel, the same types of species have been efficiently and sustainably generated in the hydrogen production field with a proton as the hydride source (Figure 1B). ${ }^{2}$ For example, hydrogen evolution via electrochemically generated Co-H species was known as early as $1985 .{ }^{21}$ The field was dormant for over two decades until recently, with interest in cobalt-catalyzed electrochemical hydrogen evolution for green energy storage being the subject of a large body of studies spanning hundreds of publications. ${ }^{22}$ These robust $\mathrm{Co}-\mathrm{H}$ based processes feature high turnover numbers and have been optimized to high levels with $>90 \%$ efficiency of $\mathrm{H}_{2}$ production from simple protic systems suggesting their commercial implementation is immanent. From a mechanistic standpoint, $\mathrm{Co}-\mathrm{H}$ is formed in-situ by the protonation of low valent $\mathrm{Co}(\mathrm{I}) / \mathrm{Co}(0)$ intermediates after direct cathodic reduction. ${ }^{23,24}$ Subsequently, it can react via two different pathways to form hydrogen and regenerate the cobalt catalyst. In the first suggested mechanism, the generated $\mathrm{Co}(\mathrm{II})-\mathrm{H}$ species decomposes by proton attack and evolves hydrogen via an intermediate dihydrogen metal complex. Alternatively, $\mathrm{Co}(\mathrm{III})-\mathrm{H}$ can be reduced to $\mathrm{Co}(\mathrm{II})-$ $\mathrm{H}$ which is followed by a similar protonation step. Interestingly, the described process operates with high faradaic efficiency in aqueous or non-aqueous mediums and various types of proton sources, such as water, TFA, TsOH, and alcohols. Amongst the many cobalt complexes enlisted, many do not require complex ligand architectures with simple bipyridyl or porphyrins derivatives performing robustly. ${ }^{25,26}$

Inspired by the well-established Co-electrocatalytic hydrogen evolution chemistry precedent outlined above, disclosed herein (Figure 1B) is a set of chemoselective, tunable electrochemical HAT (eHAT) protocols free of chemical reductants and oxidants (e.g. silanes, peroxides) or rigorous experimental protocols (moisture tolerant, no glove-box). Thus, a versatile range of tunable reactivities with alkenes and alkynes - such as isomerization, selective reduction, and hydrofunctionalization (Figure 1C)-can be realized with unmatched efficiency and chemoselectivity beyond that observed under purely chemical conditions. In support of these claims, this electrochemically-enabled reactivity is benchmarked with several of the most popular and recently disclosed chemical methods. Additionally, in-depth mechanistic analysis using cyclic voltammetry, UV-VIS spectroelectrochemistry, computation, and kinetics provides insight into e-HAT and rationalizes the observed selectivity. Finally, the scalability of this process is demonstrated in both batch and flow [gram-centigram (0.05-0.8 mole) scale]. 
As a proof of concept, alkene isomerization was selected as a model transformation since only a substoichiometric amount of $\mathrm{Co}-\mathrm{H}$ is required for the reaction to proceed with complete conversion. ${ }^{27}$ Alkene 1 was selected for the initial optimization of the electrochemical HAT (e-HAT). Trial runs using the literature precedents for classical HAT isomerization with alkene 1 provided poor conversion to the desired product. For example, the use of $50 \mathrm{~mol} \%$ of silane and $10 \mathrm{~mol} \%$ of cobalt catalyst in benzene gives $29 \%$ yield of the desired product and $7 \%$ of other chain-walking isomers (entry 1, Shenvi's protocol). ${ }^{28}$ The method of Norton, which relies on a high pressure of hydrogen gas, delivered only traces of product (entry 2). ${ }^{29}$ First forays into e-HAT isomerization followed the guiding principles ${ }^{29}$ from prior findings in electrochemistry ${ }^{31,32}$ and HAT chemistry ${ }^{33}$ to aid in the selection of proper ligands, cathodic materials and proton sources. An abbreviated summary of over 200 experiments is depicted in Figure 1D (see SI for an extensive list). First, the cobalt catalyst screening (entries 3-6) revealed that $\mathrm{CoBr}_{2}$ (glyme) was optimal, resulting in efficient $\mathrm{Co}-\mathrm{H}$ generation with the highest yield for the alkene isomerization. However, the mass balance of these reactions consisted of a mixture of chain-walking and reduction byproducts. Thus, ligand screening was performed to cleanly obtain the desired single isomerization product (entries 7-10). Although 4,4'-dimethoxy-bipyridine cleanly afforded the desired selective 1position isomerization, the conversion was low ( $38 \%$ yield $+33 \%$ recovered 1 , entry 10$)$. In order to improve the conversion while retaining the ligand-controlled selectivity, various proton sources within a wide range of $p \mathrm{~K}_{\mathrm{a}}$ were explored (entries 10-13); the use of inexpensive triethylamine hydrotetrafluoroborate ( 3 equiv., entry 13 ) as a proton source emerged as optimum, providing the desired product $\mathbf{2}$ in $\mathbf{7 2} \%$ isolated yield. This unique proton source was employed due to its ability to function as a supporting electrolyte as well; its inclusion was crucial to the reproducibility and robustness of the reaction. The final set of e-HAT conditions tolerates moisture and leads to completion with catalytic amounts of electricity $(0.5 \mathrm{~F} / \mathrm{mol}$, entry 14$)$, and can be set up in minutes using a simple undivided cell and a commercial potentiostat. Interestingly, similar reactivity was not observed when conventional reductants, such as zinc and manganese, were used (entries 15-16). Of all cathodes evaluated, tin, Nifoam, glassy-carbon, and stainless steel could be employed, but a tin cathode gave the highest yield across a broad range of substrates (see $\mathrm{SI}$ ). 


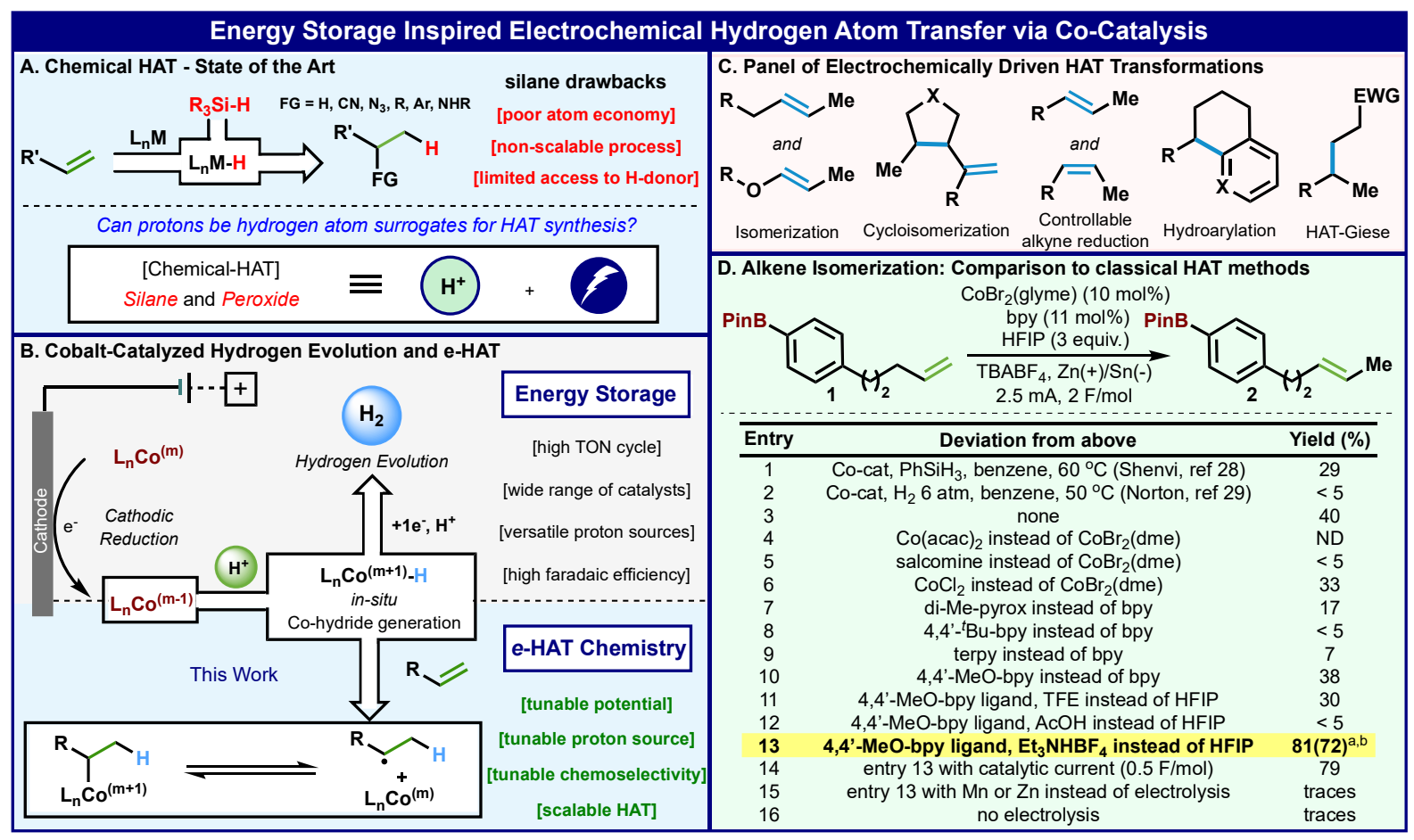

Figure 1: A) Classical HAT chemistry. B) Cobalt-catalyzed $\mathrm{H}_{2}$ evolution and e-HAT. C) e-HAT transformation. D) Optimization table of alkene isomerization. ${ }^{a}$ isolated yield ${ }^{b} E / Z$ ratio $=4 / 1$.

With these results in hand, the scope of the e-HAT isomerization of monosubstituted olefins was investigated (Table 1A). A wide range of functionalities was tolerated, including free and protected amines $(\mathbf{3}, \mathbf{6})$, anilines (5), amides (4), lactams (7), alcohols (13), and nitriles (14) with over $80 \%$ yield on average. In addition, the e-HAT isomerization exhibited a broad scope across a range of different arenes. Under optimized conditions, alkenes were isomerized in the presence of a pyridine (8), a thiophene (9), an electron-deficient indole (10), a redox-active aryl bromide (11), and an aryl-BPin (2). Remarkably, this method can deliver the isomerization of an allylic ether to the corresponding enol ether adduct (16). Terminal disubstituted olefins were however, untouched by the bipyridine complex (Conditions $\boldsymbol{A}$ ). As a result, another round of optimization was conducted revealing that Co" $(t-B u, t-B u-c y c l o h e x y l s a l e n)$ (Co(salen)-1) could be employed to exclusively isomerize such olefins to the thermodynamic trisubstituted alkenes by using HFIP (hexafluoroisopropanol) as the proton source and a Ni-foam cathode in acetone (Conditions B, see SI for optimization). As e-HAT relies on the in-situ formation of a cobalthydride, chemoselective reactions are thus possible simply by tuning conditions. A similarly broad scope was observed for this isomerization as well (17-25). The ability to achieve olefin isomerizations in the presence of free phenols, pyridines, anilines, nitriles, and epoxides is without precedent. In addition, the generation of $\beta, \gamma$-unsaturated amides has not been previously reported by isomerization methods, presumably due to a tendency to isomerize into conjugation.

Given the radical nature of intermediates in HAT-based reactions, Conditions $B$ not surprisingly initiated intramolecular radical cyclizations of dienes to form new $\mathrm{C}-\mathrm{C}$ bonds via cycloisomerization. Accordingly, methallyl prenyl malonate can undergo intramolecular cycloisomerization to yield the corresponding trisubstituted cyclopentane (26) in a high yield with no isomerization side products. The 
malonate can be exchanged by an ether (27) or an amine (28) without compromising the high efficiency of the transformation. Endocyclic alkenes can be similarly used as effective cyclization partners to form the cis five-six bicyclic systems with high diastereoselectivity (29). Even a cyclic enol ether can be used as the radical acceptor (31). As shown in Table 1, the reaction shows high efficiency for 5-membered ring formation but is less suitable for formation of 6-membered rings (30), which gave only $50 \%$ yield along with a linear isomerization side-product.

Table 1: Scope of $e$-HAT isomerization

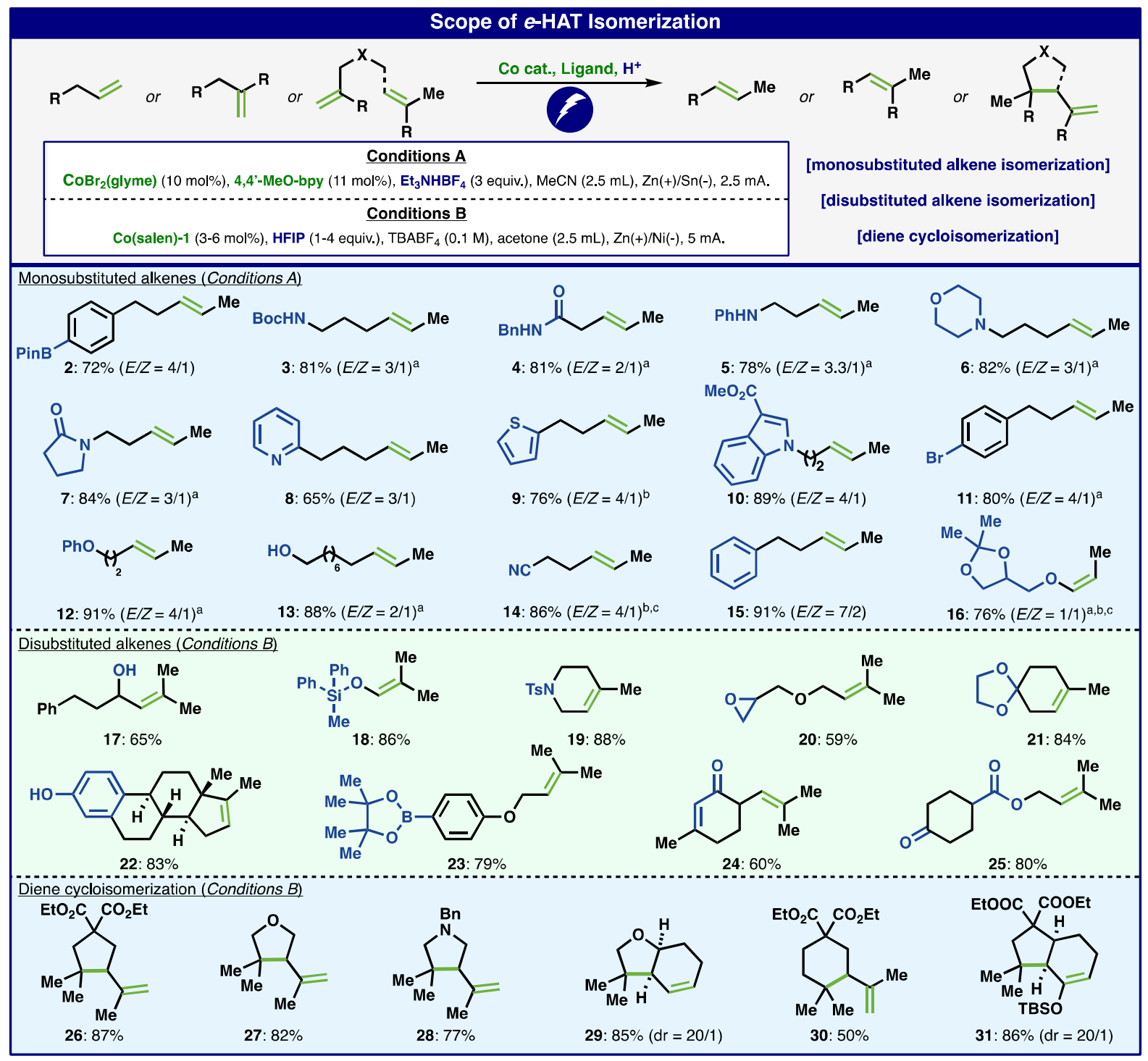

${ }^{a} \mathrm{Mg}$ was used as an anode. ${ }^{\mathrm{b}} 20$ mol\% of catalyst was used. ${ }^{\mathrm{c}} \mathrm{NMR}$ yield.

The previously discussed isomerization reactions are net-redox-neutral transformations. Therefore, a substoichiometric amount of cobalt hydride is needed to proceed efficiently since the active catalyst is regenerated during the reaction pathway (vide infra). Alternatively, cobalt-hydride chemistry 
can be used to reduce unsaturated systems. Such reactions, by definition, will require "stoichiometric" electrons to be added with the right tuning of the proton source and cobalt complex to achieve the desired transformation. As a proof of concept, using e-HAT logic, a new e-HAT set of conditions for Z-selective alkyne semi-reduction using HFIP as the hydride surrogate was developed. To place this into context, the most frequently employed reagents (i.e. outside a glove-box) to achieve such a reaction on unactivated (non-conjugated) alkynes involve the use of $\mathrm{Pd}$ (Lindlar) catalysis and diimide. For such selectivity, the 6,6'-dimethyl-bpy ligand combined with $\mathrm{CoBr}_{2}$ revealed the best reactivity delivering high Z-selectivity and minimal over-reduction (Conditions $\boldsymbol{C}$, see $\mathrm{SI}$ for optimization). With this new set of conditions in hand, a range of substituted alkynes could be reduced with e-HAT to provide Z-alkenes in good yield (Table 2) rapidly. Boc-protected amines (32), pyridines (33), ethers (34), free and silyl-protected alcohols $(36,41)$, aryl chlorides (37), aryl-BPin (38), carbonyls $(\mathbf{3 9}, \mathbf{4 2})$, alkenes (39), and phosphates (40) were all tolerated. The highest $Z / E$ selectivity was observed with primary-, secondary-, and tertiary carbons adjacent to the alkyne moiety. However, the process was less selective with quaternary carbons adjacent to the alkyne (see $\mathbf{4 3}$ and $\mathbf{4 4}$ ), presumably due to the increased steric hindrance surrounding the putative cobalt-alkene intermediate.

The selective reduction of monosubstituted alkenes was similarly achieved by relying on e-HAT (Conditions $\mathrm{C}$ ). Canonical hydrogenation with $\mathrm{H}_{2}$ over $\mathrm{Pd} / \mathrm{C}$ can accomplish this kind of reduction; however, the chemoselectivity of that method can be poor when competing for reductively labile functionalities are present. Accordingly, the e-HAT was tested with substrates that can be challenging with such well-established reduction protocols. Thus, olefins containing amine-diol functionalities (45), trisubstituted alkenes $(\mathbf{4 6}, \mathbf{4 7})$, Cbz-protected amines (48), benzyl-protected carboxylic acids (48), and thioanisole moieties (49), which can all be problematic under typical hydrogenation conditions, were smoothly reduced.

Table 2: Scope of e-HAT reduction 


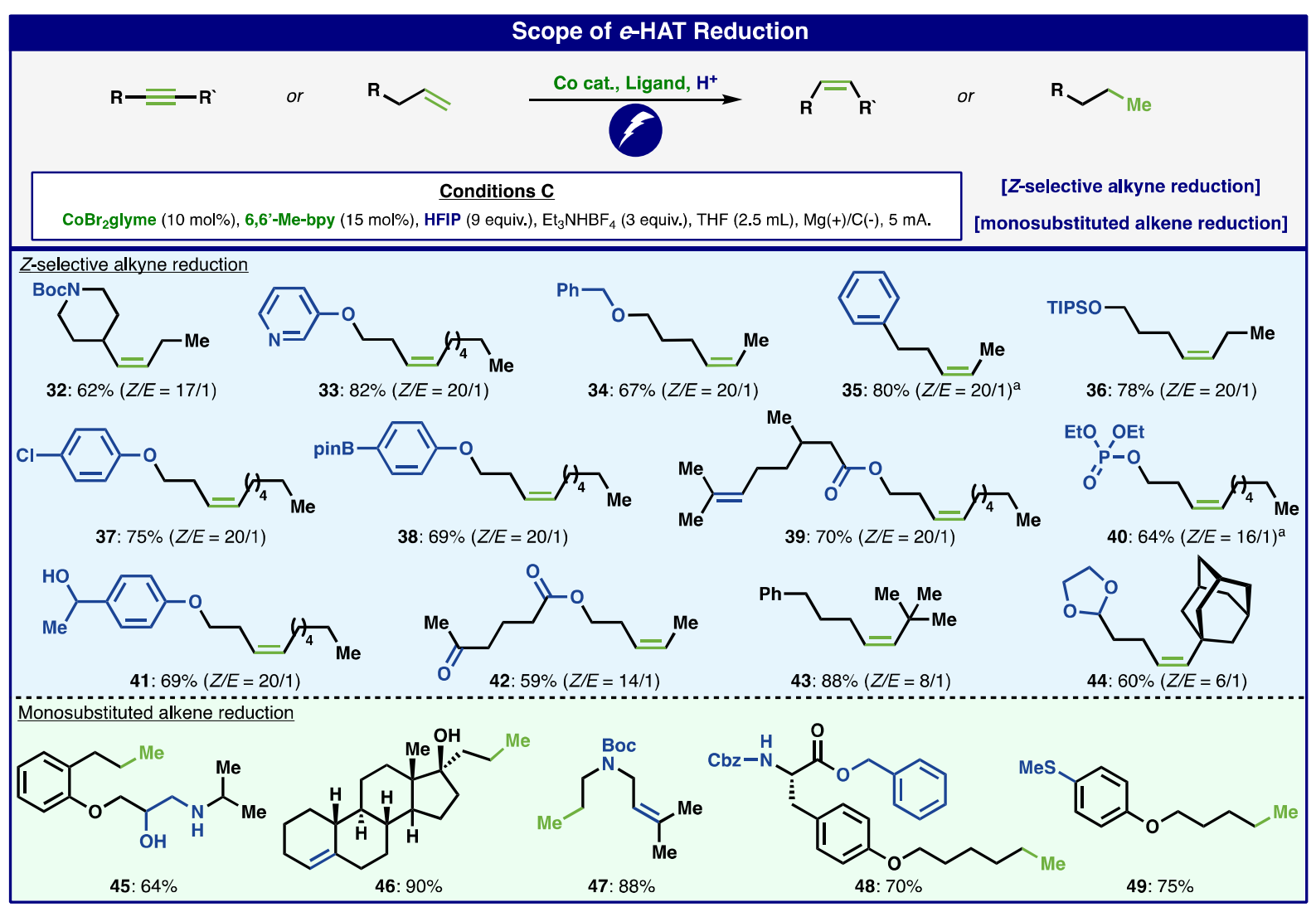

${ }^{\text {a }}$ NMR yield.

Although classical HAT chemistry has been studied for over 30 years, precise control of chemoselectivity when multiple olefins are present in a substrate has remained underdeveloped due to the complexity of tuning the hydride donor, oxidant, and catalyst. The modularity of e-HAT can potentially address such a challenge to achieve unique and useful selectivity with polyunsaturated systems and was thus pursued using the three distinct e-HAT conditions $(\boldsymbol{A}-\boldsymbol{C})$ disclosed above. Compound 50, which contains two different mono- and disubstituted terminal alkenes, was chosen as a case study (Figure 2A1). By applying Conditions $\boldsymbol{B}$ (Co(salen)-1/HFIP), the exocyclic alkene was selectively isomerized to form the thermodynamically favoured trisubstituted alkene product $\mathbf{5 1}$ in $\mathbf{5 9 \%}$ yield. In contrast, exposing compound $\mathbf{5 0}$ to Conditions $\boldsymbol{A}\left(\mathrm{CoBr}_{2} / 4,4^{\prime}-\mathrm{MeO}-\mathrm{bpy} / \mathrm{Et}_{3} \mathrm{NHBF}_{4}\right)$ led to the formation of disubstituted alkene 52 in $92 \%$ yield with an $E: Z$ isomeric ratio of 3:1. To date, such selectivity has not been reported using canonical HAT reactivity. Similarly, evaluation of the e-HAT alkene reduction displayed exquisite selectivity towards terminal monosubstituted alkenes. Triene $\mathbf{5 3}$ was subjected to Conditions $\boldsymbol{C}$ which selectively reduced the desired alkene - over the 1,1- disubstituted and endocyclic olefins - in $95 \%$ isolated yield (Figure 2A-3). Likewise, diene $\mathbf{5 5}$ was also examined under the same conditions and exhibited similar chemoselectivity towards the monosubstituted olefin in the presence of an internal disubstituted alkene to furnish 56 in 81\% yield. As there are numerous alkene reduction methods available, the results of e-HAT could be placed into context through a direct comparison with the prior art. Using $\mathrm{H}_{2}$ with $\mathrm{Pd} / \mathrm{C}$, dimide, PET (Co-Salen with $\mathrm{Ru}$ catalyst and light) ${ }^{34}$, and $\mathrm{Co} / \mathrm{H}^{+}$-based ${ }^{35}$ systems resulted in an inseparable mixture of reduced or isomerized products (see SI). 
A different and useful case of $e$-HAT chemoselectivity is exemplified with the isomerization of 1,1disubstituted olefins to afford the less hindered isomerization products (Figure 2A-2). In the case of compound 57, the double bond migration process has essentially two unpredictable directions to form different trisubstituted alkenes. By applying the Co(salen)-1/HFIP conditions with compound 57, the regioselectivity control exclusively furnished product $\mathbf{5 8}$ (89\%). Such regioselectivity could be explained through steric discrimination as guided by the catalyst. To strengthen this hypothesis, compound $\mathbf{5 9}$ was also tested under the same conditions; although there is a driving force to form the tetrasubstituted thermodynamic alkene, the kinetically favored trisubstituted alkene was identified as the major product with a 7:1 isomeric ratio.

Next, the scalability of e-HAT was evaluated in both batch and recycle flow on three different transformations; isomerization, alkyne reduction, and cycloisomerization (Figure 2B). The isomerization reaction with Conditions $\boldsymbol{A}$ was tested with compound $\mathbf{6 1}$ on a $1 \mathrm{~g}$ scale using the commercial Vapourtec ion recycle flow system (Figure 2B-1). By keeping the same parameters with $0.8 \mathrm{~mA} / \mathrm{cm}^{2}$ current density and $1 \mathrm{ml} / \mathrm{min}$ flow rate, the product was obtained in $92 \%$ yield. In a similar fashion, the alkyne reduction was demonstrated on a $10 \mathrm{~g}$ scale using a batch setup to achieve a complete conversion of $\mathbf{6 4}$ and obtain the desired product (65) with a $76 \%$ isolated yield (11:1 of $E: Z$ isomeric ratio, Figure $2 \mathrm{~B}-2$ ). Finally, the cycloisomerization reaction with Conditions $B$ was conducted on a $100 \mathrm{~g}$ scale with compound $\mathbf{6 1}$ using a recycle flow apparatus containing four reaction cells (Figure 2B-3). After optimization, 100 grams of $\mathbf{6 1}$ were successfully converted to product 27 by keeping the same current value of $2 \mathrm{~mA} / \mathrm{cm}^{2}$ (compared to $0.2 \mathrm{mmol}$ scale) with a catalytic current of $0.6 \mathrm{~F} / \mathrm{mol}$ to achieve complete conversion ( $73 \%$ isolated yield).

The direct hydrofunctionalization of saturated systems represents an area where HAT-based transformations have found extensive application in synthesis. ${ }^{7}$ Accordingly, the e-HAT could be implemented under a universal set of conditions to achieve a wide range of classical HAT reactivities (Figure 2C). By simply using a slight modification of Conditions B, intramolecular HAT-Giese (67), hydroarylation (71), hydropyridination (73), retroisomerization of alkene-conjugated strained-rings (75), and deallylation as a general deprotection method (77). For the intermolecular HAT-Giese with ethyl acrylate to form a quaternary carbon (50\%), a modification of Conditions B was employed wherein 2 mol\% of $\mathrm{Co}$ (tetramethyl-ethanediyl salen) (Co(salen)-2) enabled the reaction to proceed, whereas no product was observed with Co(salen)-1. This phenomenon might be explained by the efficient formation of the carbon-centred radical instead of the cobalt-carbon bond with Co(salen)- 2 due to geometrical differences in the cobalt precatalysts (see SI for mechanistic rationale). ${ }^{36}$

As a testament to the unique tunability of e-HAT, a challenging trans-selective alkyne semireduction was achieved using Conditions $B$ by slightly modifying the amount of HFIP to 8 equivalents and passing through $5 \mathrm{~F} / \mathrm{mol}$ of electricity. Alkyne $\mathbf{7 9}$ was therefore reduced to the corresponding trans-alkene in $60 \%$ isolated yield and high geometric selectivity $(7: 1-E / Z)$. Interestingly, by performing the analogous controls with HAT chemistry using silane and the same Co(salen)-2 complex, we found that such reactivity failed to translate, once again highlighting a unique feature of the e-HAT platform. The direct, $E$-selective reduction of alkynes can currently only be accomplished using either harsh Birch-like conditions $\left(\mathrm{Li} / \mathrm{NH}_{3}\right)^{37}$ or by enlisting an expensive Ru-based catalysis system. ${ }^{38}$ 


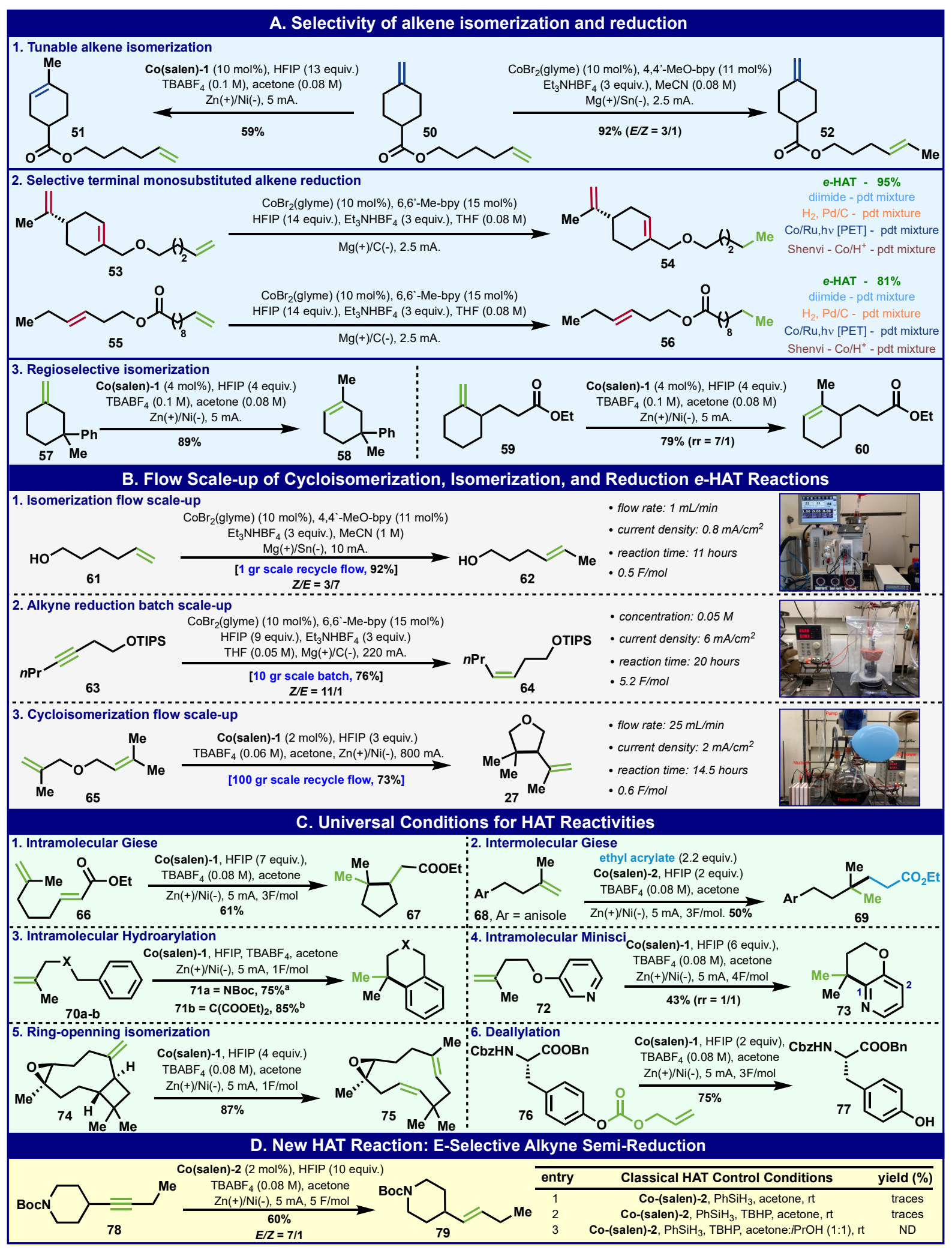


Figure 2: Selectivity, scalability, and reactivity of e-HAT. ${ }^{a} \mathrm{HFIP}$ (7 equiv.), $\mathrm{TBABF}_{4}(0.08 \mathrm{M}){ }^{\mathrm{b}} \mathrm{HFIP}$ (2 equiv.), $\mathrm{TBABF}_{4}(0.08 \mathrm{M})$.

To further understand the e-HAT processes and support the critical steps of the proposed mechanism, cyclic voltammetry (CV), UV-vis spectroelectrochemistry, DFT computations, and kinetic analysis were employed. First, we investigated the cyclic voltammetry profiles of the Co(salen)-1 isomerization system. As shown in Figure 3B-1, the CV profile of Co(Salen)-1 at $50 \mathrm{mV} / \mathrm{s}$ showed a reversible redox peak at $-1.98 \mathrm{~V}$ corresponds to the $\mathrm{Co}(\mathrm{II} / \mathrm{I})$ redox couple (step A). The addition of HFIP to the Co(Salen)-1 solution results in the observation of catalytic current. This is expected to arise from an ECEC $_{\text {cat }}$ process corresponding to the generation of $\mathrm{H}_{2} \cdot{ }^{23,24}$ Such an observation is suggestive of in-situ protonation of the low-valent $\mathrm{Co}(\mathrm{I})$ to generate the $\mathrm{Co}(\mathrm{III})$-H (step B). The addition of both HFIP and alkene to the Co(salen)-1 solution (Conditions B) results in a slight dampening of the catalytic current. This could indicate that the alkene is reacting with the $\mathrm{Co}-\mathrm{H}$ species to catalyze the isomerization reaction, diverting the catalyst from the $\mathrm{H}_{2}$ generation cycle (step C). To further support the proposed mechanism of the Co(salen)-1 system, we sought to observe the respective catalyst resting states with in-situ UV-vis spectroelectrochemistry (Figure 3B-2). Accordingly, we examined the spectroelectrochemical properties of the Co(salen)-1 under the olefin isomerization reaction mixture. Application of a stepwise reducing potential to a $\mathrm{Co}(\mathrm{II})$-salen solution found modest decreases in absorbance for the electronic transitions at $416 \mathrm{~nm}$ and $492 \mathrm{~nm}$, which could have resulted from either the direct reduction of the metal center to a $\mathrm{Co}(\mathrm{I})$-salen (Step A) or redox non-innocence of the salen backbone (Figure 3B-2, and see SI). Reduction of Co(salen)-1 at $-2.03 \mathrm{~V}$ in the presence of HFIP led to the complete disappearance of the Co(II)-salen electronic spectral features and appearance of a single UV-vis absorbance at $375 \mathrm{~nm}$. Based on our previous observations, upon application of reducing potentials, we speculate that this new spectral feature at $375 \mathrm{~nm}$ could arise from the presence of a Co-hydride catalyst resting state (Step B), a commonly invoked intermediate within the field of Co-promoted HER catalysis. ${ }^{39,40}$ Intriguingly, spectroelectrochemical studies of the Co(salen)-1 in the presence of HFIP and alkene lead to the same absorbance feature at $375 \mathrm{~nm}$ during active electrocatalysis. Presumably this putative Co-hydride could be competent for both Co-promoted HER as well as Co-catalyzed olefin isomerization, thus appearing in both spectroelectrochemical experiments as a plausible catalyst resting state.

To investigate the $\mathrm{CoBr}_{2} / 4,4^{\prime}$-dimethoxybipyridine isomerization system (Conditions $\boldsymbol{A}$ ), the ligation state of the active cobalt catalyst was first identified (Figure 3B-3). A 1:1 mixture of $\mathrm{CoBr}_{2}$ and 4,4'dimethoxybipyridine resulted in a CV profile with two distinct reduction peaks for $\mathrm{Co}$ (II) to $\mathrm{Co}$ (I) (Figure $3 \mathrm{~A}$, step A). These peaks were preliminarily assigned as the bisligated $\mathrm{L}_{2} \mathrm{CoBr}_{2}$ (which overlaps with unligated $\mathrm{CoBr}_{2}$ ) for the less negative reduction peak and the monoligated $\mathrm{L}_{1} \mathrm{CoBr}_{2}$ for the more negative reduction peak. This assignment is consistent with previous studies showing that the reduction potential of bisligated cobalt complexes is in general, less negative than its corresponding monoligated species. ${ }^{41}$ Next, CV studies were performed with adding concentration of 4,4'-dimethoxybipyridine. At 1:1.5 Co/ligand ratio, both reduction peaks increased. The addition of more ligand caused the peak assigned as monoligated complex to decrease. Due to the overlapping peak on the reduction wave associated with the bisligated system, the oxidation wave was examined (since the reduction of unligated $\mathrm{CoBr}_{2}$ is irreversible, see $\mathrm{SI}$ )' wherein an increased oxidation peak was observed upon increasing ligand concentration beyond 1.5 equiv. These studies provide evidence for the preliminary assignment outlined above. Next, the effect of the proton source and the alkene substrate on the CV behavior of the Cobalt 
catalyst was studied. Using a 1:2 mM ratio of $\mathrm{CoBr}_{2} /$ ligand where both the mono and bisligated species were present, the effect of adding $1 \mathrm{mM} \mathrm{Et}_{3} \mathrm{NHBF}_{4}$ was investigated (Figure 3B-4). A significant increase in peak current was observed for the monoligated cobalt complex, and a negligible change was observed for the bisligated species. These denote that the monoligated cobalt complex is active towards reaction with $\mathrm{Et}_{3} \mathrm{NHBF}_{4}$ to generate Co-hydride intermediate (Figure 3A, step B). The addition of monosubstituted alkene to this solution resulted in a catalytic current indicative of a chemical reaction with alkene that regenerates the active cobalt catalyst. We therefore propose this as a series of chemical steps for the isomerization of alkenes (Figure $3 \mathrm{~A}$, steps $\mathrm{C}$ to $\mathrm{E}$ ).

DFT computational analysis provided additional evidence for the proposed mechanism of olefin isomerization. Based on the divergent reactivity of the two complexes throughout the synthetic studies, it stands to reason that the salen derived catalysts operate with a different general mechanism than that of the bipyridine system. To explore this, the bond dissociation energies of various possible intermediates in such isomerization processes were calculated for both systems. According to the previous analysis, the reaction is initiated via $\mathrm{Co}-\mathrm{H}$ generation, followed by its insertion into the olefin. The alkyl-cobalt intermediates of salen ligands contain weak $\mathrm{Co}-\mathrm{C}$ bonds $(27 \mathrm{kcal} / \mathrm{mol}$ for secondary carbon, $20 \mathrm{kcal} / \mathrm{mol}$ for tertiary carbon, Figure 3B-5) that resemble diradicals. ${ }^{42}$ Accordingly, we propose that steps $\mathrm{C}$ and $\mathrm{E}$ (Figure $3 \mathrm{~A}$ ) proceed via $\mathrm{H}$-atom transfer with Co(salen)-1 system. Similarly, Figure 3B-6 shows that the strength of the $\mathrm{Co}-\mathrm{H}$ bond in the $\mathrm{Co}($ salen)-hydride complex indeed matches the $\mathrm{C}-\mathrm{H}$ bonds of the substrate, which further support the thermodynamic favorability of the HAT pathway. ${ }^{34}$ In the case of the Co-bipyridine system (Conditions $A$ ), the strong $\mathrm{Co}-\mathrm{C}$ bonds of the putative intermediates (Figure 3B-6) are perhaps more consistent with an organometallic process of inner-sphere migratory insertion and $\beta$ hydride elimination. Consistent with this conclusion, the bipyridine Co-hydride species potentially involved in an HAT version of step $\mathrm{C}$ contain $\mathrm{Co}-\mathrm{H}$ bonds much stronger than the substrates $\mathrm{C}-\mathrm{H}$ bonds ( $\mathrm{Co}^{\prime}$ and $\mathrm{Co}$ ", Figure 3B-5) while those potentially involved in step $\mathrm{E}$ contain $\mathrm{Co}-\mathrm{H}$ bonds much weaker than the substrate's $\mathrm{C}-\mathrm{H}$ bonds (Co'I', Figure 3B-5). Both assertions point to a thermodynamically unfavorable HAT pathway in the presence of bipyridine ligands.

Kinetic studies carried out on the isomerization of monosubstituted alkenes 61 and 5-phenyl-1pentene (15a) using the Co-bipyridine system (Conditions $\boldsymbol{A}$ ) and the cycloisomerization of $\mathbf{3 0}$ using the Co(salen)-1 system (Conditions $B$ ) revealed intriguing differences between the two cases (Figure 3B-7). For Conditions $B$, the reaction exhibits first order kinetics in both [substrate] and [Co(salen)-1], while for Conditions $A$, the reaction is zero order in both substrate and Co-bipyridine concentrations. The reaction rate under Conditions $\boldsymbol{A}$ is not influenced by current, while under Conditions $\boldsymbol{B}$, the rate increases proportionally with increasing current. Neither case is influenced by the concentration of the proton source. Neither system requires $1 \mathrm{~F} / \mathrm{mol}$ substrate reacted (dashed gray line in left and center plots in Figure 3B-7), and thus both are catalytic in current. Turnover in the cycloisomerization reaction using Co(salen)-1 continues, albeit more slowly, even after the current is stopped (right plot in Figure 3B-7). Reactions using co-bipyridine do not proceed further when current is stopped.

Both catalyst systems exhibit an induction period, the length of which decreases with increasing current (middle plot in Figure 3B-7). For Co(salen)-1, the induction period is unaffected by increasing catalyst concentration. By contrast, in the Co-bipyridine system, the length of the induction period increases as catalyst concentration increases. The induction period for Co-bipyridine could be removed by pre-activating the catalyst with $0.12 \mathrm{~F} / \mathrm{mol}$ electrolysis. Deposition of $\mathrm{Co}$ on the electrode was also inferred by CV experiments in the absence of substrate. These observations may help to explain the 
unusual finding of zero-order kinetics in [Co] in the Co-bipyridine case; the active catalyst may be a surface Co species. Further studies are underway to probe these effects.

The Z-selective semi-reduction of alkyne using $\mathrm{CoBr}_{2} / 6,6^{\prime}$-dimethylbipyridine (Conditions $\boldsymbol{C}$ ) was also investigated. Similarly, CV studies with increasing concentration of ligand were also performed (Figure 2B-8). With such a system, no distinct peak separation was observed at 1:1 ratio of $\mathrm{CoBr}_{2} /$ ligand. However, upon the increasing concentration of the ligand, a notable cathodic peak shift was observed where the reduction peak potential becomes less negative as a function of increasing ligand concentration. The shifted regions were labeled as predominantly one-electron reduction of monoligated and bisligated $\mathrm{Co}(\mathrm{II})$, respectively (Figure 3A, step A). Using a mixture of 1:1.5 ratio of $\mathrm{CoBr}_{2} /$ ligand (predominantly monoligated, and similar to the ratio in the reaction conditions), the effect of HFIP and alkyne addition (Figure 3B-9) was studied. The addition of 1 equivalent of HFIP showed a shift in the cathodic peak, denoting a chemical step that generates a new electroactive cobalt intermediate proposed as $\mathrm{Co}(\mathrm{III})-\mathrm{H}$ (Figure $3 \mathrm{~A}$, step B). The addition of alkyne to this mixture resulted in an increase in cathodic peak current with reduction potential similar to the active catalyst. This denotes a chemical reaction of the cobalt-hydride intermediate with the alkyne accompanied by regeneration of the monoligated cobalt(II) catalyst (Figure 3A, steps $\mathrm{F}$ to $\mathrm{H}$ ). Computational analysis corroborated this proposed mechanism of proton migration from HFIP to the coordinated alkyne (Figure 3B-10, step G) as it demonstrated feasible energetics. The barrier for such a process is notably low $\left(\Delta G^{\ddagger}=6.2 \mathrm{kcal} / \mathrm{mol}\right)$ as it proceeds through a completely concerted mechanism (see $\mathrm{SI}$ for details). These values provide support for step $\mathrm{G}$ of the alkyne reduction catalytic cycle. 


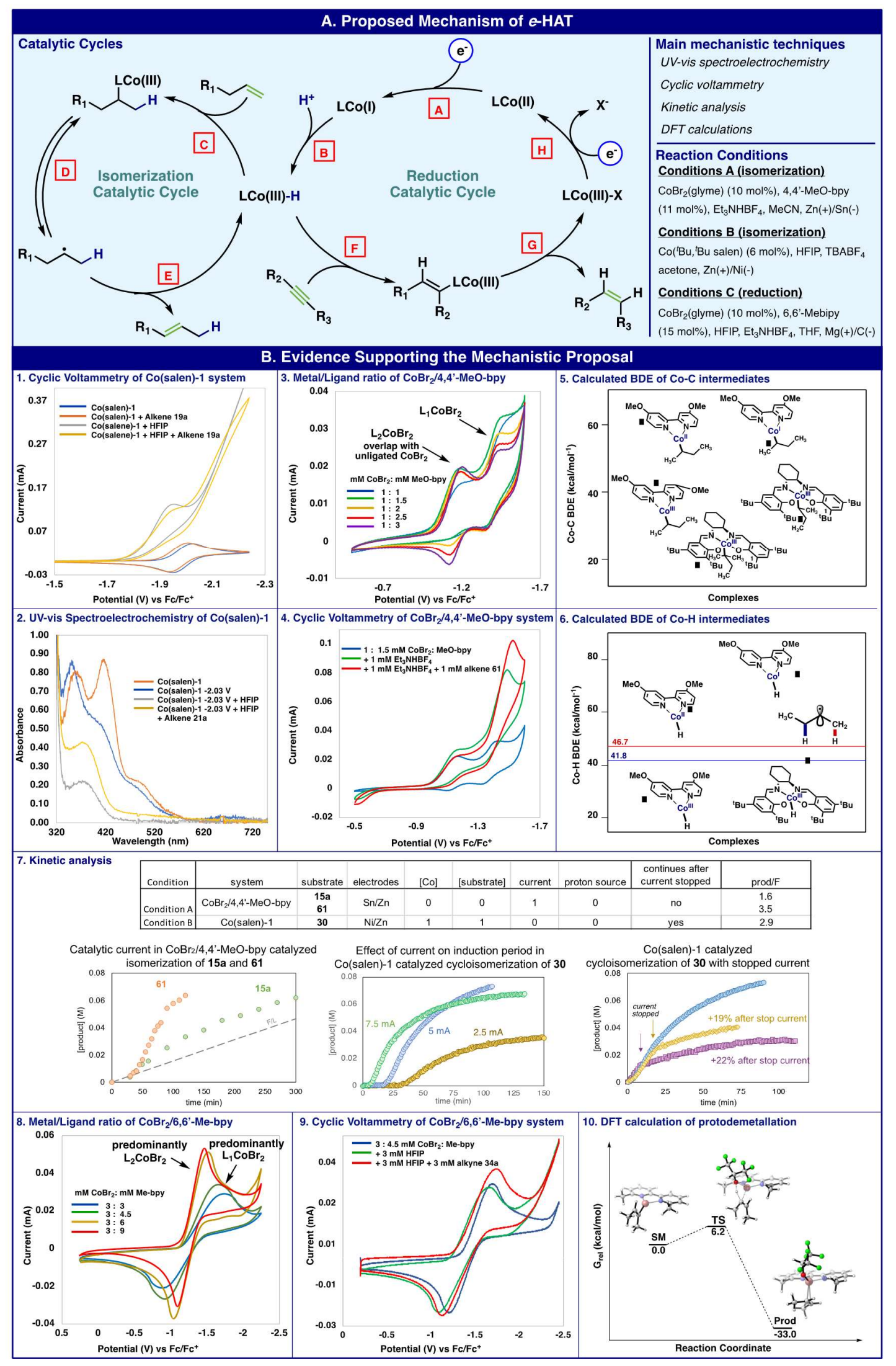


Figure 3: (A) Proposed Mechanism pathway of e-HAT. (B) Evidence supporting the mechamstic proposal using cyclic voltammetry, UV-vis spectroelectrochemistry, kinetic analysis, BDE and DFT calculations.

\section{Data availability}

The data that support the findings of this study are available from the corresponding authors upon reasonable request.

\section{Acknowledgement}

This work was supported by the NSF Center for Synthetic Organic Electrochemistry, CHE-2002158.. S.G. thanks the Council for Higher Education, Fulbright Israel and Yad Hanadiv for the generous fellowships. A.B. thanks the Austrian Science Fund (FWF) for an Erwin Schrödinger Fellowship (J 4452-N). H.-J.Z. thanks the SIOC fellowship. C. A. M. thanks the National Institute of General Medical Sciences of the National Institutes of Health (K99GM140249). The authors are grateful to D.-H. Huang and L. Pasternack (Scripps Research) for assistance with the NMR spectroscopy, to J. Chen, B. Sanchez and E. Sturgell (Scripps Automated Synthesis Facility) for assistance with HPLC, high-resolution mass spectroscopy and LCMS. We also thank Dr Y. Kawamata, Dr. K.X. Rodriguez and C. Bi for helpful advice and suggestions.

References:

1. Norton, J. R., \& Sowa, J. Introduction: metal hydrides. Chem. Rev. 116, 8315-8317 (2016).

2. Luo, G. G. et al. Recent progress in ligand-centered homogeneous electrocatalysts for hydrogen evolution reaction. Inorg. Chem. Front. 6, 343-354 (2019).

3. Margarit, C. G., Asimow, N. G., Thorarinsdottir, A. E., Costentin, C., \& Nocera, D. G. Impactful Role of Cocatalysts on Molecular Electrocatalytic Hydrogen Production. ACS Catal. 11, 45614567 (2021).

4. Shevick, S. L. et al. Catalytic hydrogen atom transfer to alkenes: a roadmap for metal hydrides and radicals. Chem. Sci. 11, 12401-12422 (2020).

5. Armstrong, K. C., \& Waymouth, R. M. Electroreduction of Benzaldehyde with a Metal-Ligand Bifunctional Hydroxycyclopentadienyl Molybdenum (II) Hydride. Organometallics, 39, 4415-4419 (2020).

6. Vesborg, P. C., Seger, B., \& Chorkendorff, I. B. Recent development in hydrogen evolution reaction catalysts and their practical implementation. J. Phys. Chem. 6, 951-957 (2015).

7. Crossley, S. W., Obradors, C., Martinez, R. M., \& Shenvi, R. A. Mn-, Fe-, and Co-catalyzed radical hydrofunctionalizations of olefins. Chem. Rev. 116, 8912-9000 (2016).

8. Wiedner, E. S. et al. Thermodynamic hydricity of transition metal hydrides. Chem. Rev. 116, 8655-8692 (2016).

9. Hu, Y., Shaw, A. P., Estes, D. P., \& Norton, J. R. (2016). Transition-metal hydride radical cations. Chem. Rev. 116, 8427-8462. 
10. Eisenberg, D. C., \& Norton, J. R. Hydrogen-atom transfer reactions of transition-metal hydrides. Isr. J. Chem 31, 55-66 (1991).

11. Puls, F., Linke, P., Kataeva, O., \& Knölker, H. J. Iron-Catalyzed Wacker-type Oxidation of Olefins at Room Temperature with 1, 3-Diketones or Neocuproine as Ligands. Angew. Chem, Int. Ed. (2021).

12. Isayama, S., \& Mukaiyama, T. A New Method for Preparation of Alcohols from Olefins with Molecular Oxygen and Phenylsilane by the Use of Bis (acetylacetonato) cobalt (II). Chem. Lett. 18, 1071-1074 (1989).

13. Gui, J. et al. Practical olefin hydroamination with nitroarenes. Science, 348, 886-891 (2015).

14. Lo, J. C., Gui, J., Yabe, Y., Pan, C. M., \& Baran, P. S. Functionalized olefin cross-coupling to construct carbon-carbon bonds. Nature, 516, 343-348 (2014).

15. Gaspar, B., \& Carreira, E. M. Mild cobalt-catalyzed hydrocyanation of olefins with tosyl cyanide. Angew. Chem. In. Ed., 46(24), 4519-4522 (2007).

16. Shigehisa, H., Aoki, T., Yamaguchi, S., Shimizu, N., \& Hiroya, K. Hydroalkoxylation of unactivated olefins with carbon radicals and carbocation species as key intermediates. J. Am. Chem. Soc. 135(28), 10306-10309 (2013).

17. Leggans, E. K., Barker, T. J., Duncan, K. K., \& Boger, D. L. Iron (III)/NaBH4-mediated additions to unactivated alkenes: synthesis of novel 20'-vinblastine analogues. Org. Lett. 14(6), 1428-1431 (2012).

18. Wells, A. S. On the perils of unexpected silane generation. Org. Process Res. Dev. 14, 484-484 (2010).

19. Yu, K., Yao, F., Zeng, Q., Xie, H., \& Ding, H. Asymmetric Total Syntheses of (+)-Davisinol and (+)18-Benzoyldavisinol: A HAT-Initiated Transannular Redox Radical Approach. J Am. Chem. Soc. (2021). https://doi.org/10.1021/jacs.1c05703

20. Godfrey, N. A., Schatz, D. J., \& Pronin, S. V. Twelve-step asymmetric synthesis of (-)-nodulisporic acid C. J. Am. Chem. Soc. 140, 12770-12774 (2018).

21. Kellett, R. M., \& Spiro, T. G. Cobalt (I) porphyrin catalysts of hydrogen production from water. Inorg. Chem. 24, 2373-2377 (1985).

22. Zhang, W., Cui, L., \& Liu, J. Recent advances in cobalt-based electrocatalysts for hydrogen and oxygen evolution reactions. J. Alloy. Compd. 821, 153542 (2020).

23. Wiedner, E. S., \& Bullock, R. M. Electrochemical detection of transient cobalt hydride intermediates of electrocatalytic hydrogen production. J. Am. Chem. Soc. 138, 8309-8318 (2016).

24. Marinescu, S. C., Winkler, J. R., \& Gray, H. B. Molecular mechanisms of cobalt-catalyzed hydrogen evolution. PNAS 109, 15127-15131 (2012).

25. Beyene, B. B., Mane, S. B., \& Hung, C. H. Electrochemical hydrogen evolution by cobalt (II) porphyrins: effects of ligand modification on catalytic activity, efficiency and overpotential. J. Electrochem. Soc. 165, 481 (2018).

26. Queyriaux, N., Jane, R. T., Massin, J., Artero, V., \& Chavarot-Kerlidou, M. Recent developments in hydrogen evolving molecular cobalt (II)-polypyridyl catalysts. Coordin. Chem. Rev. 304, 3-19 (2015).

27. Kapat, A., Sperger, T., Guven, S., \& Schoenebeck, F. E-Olefins through intramolecular radical relocation. Science 363, 391-396 (2019).

28. Crossley, S. W., Barabé, F., \& Shenvi, R. A. Simple, chemoselective, catalytic olefin isomerization. J. Am. Chem. Soc. 136, 16788-16791 (2014). 
29. Li, G. et al. Radical isomerization and cycloisomerization initiated by $\mathrm{H} \bullet$ transfer. J. Am. Chem. Soc. 138, 7698-7704 (2016).

30. Kingston, C. et al. A Survival Guide for the "Electro-curious". Acc. Chem. Res. 53, 72-83 (2019).

31. Gao, Y. et al. Electrochemical Nozaki-Hiyama-Kishi Coupling: Scope, Applications, and Mechanism. J. Am. Chem. Soc. 143, 9478-9488, (2021).

32. Gnaim, S. et al. Electrochemically driven desaturation of carbonyl compounds. Nat. Chem. 13, 367-372 (2021).

33. Lo, J. C et al. Fe-catalyzed C-C bond construction from olefins via radicals. J. Am. Chem. Soc. 139, 2484-2503 (2017).

34. Kamei, Y. et al. Silane-and peroxide-free hydrogen atom transfer hydrogenation using ascorbic acid and cobalt-photoredox dual catalysis. Nat. Commun. 12, 1-9 (2021).

35. Van der Puyl, V., McCourt, R. O., \& Shenvi, R. A. Cobalt-catalyzed alkene hydrogenation by reductive turnover. Tetrahedron Lett. 72, 153047 (2021).

36. Yin, Y. N., Ding, R. Q., Ouyang, D. C., Zhang, Q., \& Zhu, R. Highly chemoselective synthesis of hindered amides via cobalt-catalyzed intermolecular oxidative hydroamidation. Nat. Commun. 12, 1-10 (2021).

37. Benkeser, R. A., Schroll, G., \& Sauve, D. M. Reduction of organic compounds by lithium in low molecular weight amines. II. Stereochemistry. Chemical reduction of an isolated non-terminal double bond. J. Am. Chem. Soc. 77, 3378-3379 (1955).

38. Fürstner, A. trans-Hydrogenation, gem-Hydrogenation, and trans-Hydrometalation of Alkynes: An Interim Report on an Unorthodox Reactivity Paradigm. J. Am. Chem. Soc. 141, 11-24 (2018).

39. Wiedner, E. S., \& Bullock, R. M. Electrochemical detection of transient cobalt hydride intermediates of electrocatalytic hydrogen production. J. Am. Chem. Soc. 138, 8309-8318 (2016).

40. Walaijai, K., Cavill, S. A., Whitwood, A. C., Douthwaite, R. E., \& Perutz, R. N. Electrocatalytic Proton Reduction by a Cobalt (III) Hydride Complex with Phosphinopyridine PN Ligands. Inorg. Chem. 59, 18055-18067 (2020).

41. Hickey, D. P. et al. Investigating the role of ligand electronics on stabilizing electrocatalytically relevant low-valent Co (I) intermediates. J. Am. Chem. Soc. 141, 1382-1392 (2019).

42. Qi, X. J., Li, Z., Fu, Y., Guo, Q. X., \& Liu, L. anti-Spin-Delocalization Effect in Co- C Bond Dissociation Enthalpies. Organometallics 27, 2688-2698 (2008).

Table of Content

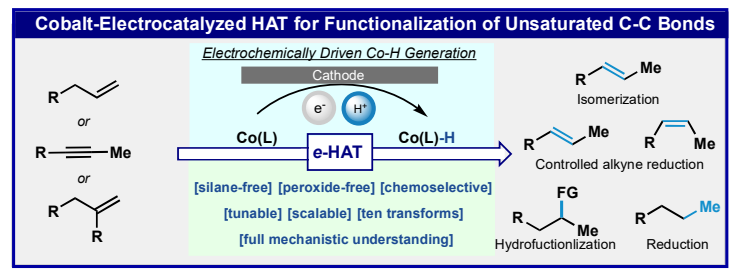

